(Physiologisches Laboratorium in Bonn.)

\title{
Herr Professor Carl Voit und die Beziehung der Athembewegungen zu dem Stoffwechsel.
}

\author{
Von
}

\section{E. Pfiuger.}

Carl Voit hat in der Zeitschrift für Biologie ${ }^{1}$ ) Prioritätsreclamationen gegen mich erhoben und sich hierin von einem meiner früheren Schüler, Hrn. Dr. Siegf ried Wolffberg ${ }^{2}$ ), der in Voit's Laboratorium später arbeitete, unterstützen lassen. Eine eingehende Widerlegung setzt eine Kritik der von Voit und Pettenk of er mit dem Respirationsapparate des Letzteren erhaltenen Resultate voraus, auf welche ich des ungeheuren Umfanges des darüber gedruckten Materiales halber an einer anderen Stelle ausführlich zurückkommen werde.

Es soll heute meine Aufgabe sein, zu zeigen, wie unberechtigt die Ansprüche Carl Voit's auf die Erkenntniss der wahren Beziehungen zwischen Stoffwechel und Athembewegungen sind.

Wenn die folgenden Erörterungen nur Prioritätsreclamationen beträfen, würde ich lieber geschwiegen haben; ich muss aber zugleich mehre wichtige Mittheilungen machen.

Nachdem durch Lord Baco von Verulam und besonders durch John Mayow, dem eigentlichen Vorläufer von Lavoisier

1) Carl Voit's Beschreibung eines Apparates zur Untersuchung der gasförmigen Ausscheidungen des Thierkörpers. Zeitschrift f. Biologie XI. pg.536.

2) Dr. S. Wolffberg, Assistenzarzt an der Krankenhaus-Abtheilung des Hrn. Prof. v. Ziemssen, Ueber den Ursprung und die Aufspeicherung des Glykogens im thierischen Organismus; Zeitschr. f. Biologie. Bd, XII. pg. 292. 
Prof. c. Voit u. d. Beziehung d. Athembewegungen zu dem Stoffwechsel. 631

die - wenn auch das Wesen nicht erschöpfende - Wahrheit erkannt worden war, dass der Lebensprocess eine durch den Sauerstoff der Luft ) bedingte langsame Verbrennung sei, gelangten die meisten Physiologen und Aerzte zu der Ansicht, dass die Lungen den Blasebälgen vergleichbar wären, die eine Schmiedeesse zu um so energischerem Brande anfachen, je stärker sie ventilirt werden. Fast überall begegnet man in der Literatur dieser Auffassung, der zufolge ein Mensch nur recht lebhaft zu athmen braucht, um auch eine energischere Wärmebildung in sich anzuregen.

Nachdem dann Lavoisier ${ }^{2}$ ) in den mit Seguin ausgeführten Untersuchungen die von Regnault und Reiset ${ }^{3}$ ) bestätigte merkwürdige Thatsache gefunden hatte, dass der thierische Verbrennungsprozess absolut derselbe bleibe, gleichgültig ob ein Thier atmosphärische Luft oder reines Sauerstoffgas athme, konnte man schon mit grosser Wahrscheinlichkeit schliessen, dass die grössere

1) John Ma yow sagte:

- Die Theilchen der sebensluft sind nicht die Luft selbst, sondern nur ein feinerer Theilderselben. Seine eigenen Worte:

-Particulae aëris igneae, vitalesque non suntipse är, sed tantum parsejus subtilior.s

Bei dem Wort ,feinerer Theil" muss man die von Mayow auf Grund eines ingeniösen Versuches aufgestellte richtige Behauptung beachten, dass der nicht athembare Theil der Luft der specifisch leichtere Theil sei. Mayow kannte die Bedeutung der Lebensluft für die Erzengung der Säuren, ja sogar des Salpeters und die Zunabme des Gewichts des brennbaren Körpers durch die Verbindung mit der Lebensluft. - Er bewies, dass Leben and Verbrennung durch die Lebensluft bedingt sind; dass diese in den Lungen von dem Blute absorbirt wird und das Blut roth macht; glaubt, dass die Lebensluft aus dem Blut nach den Organen diffundirt, wobei er die Placentarrespiration erklärte. Er erkannte, dass die Lebensluft die Muskelbewegung ermöglicht, sowie die Ursache der \&ebensfermentation a ist. So schrieb Mayow 1681 - ein Jahrhundert vor Lav oisier - so schrieb ein von der dankbaren Nachwelt selten genannter, jung verstorbener, grosser Prophet, sogar Lavoisier oft in der Erkenntniss der Respiration weit überholend.

2) Lavoisier et $\mathrm{Segu}$ in, Premier mémoire sur la respiration des animaux. Oenvres de Lavo isi er T. II. pg.694 oder Mém. de l'acad. des sciences 1789. p. 566.

3) Regnault et J. Reiset, Recherches chimiques sur la respiration des animaux des diverses classes; - Annal, de Chimie et de Physique. 3me Serie. T. XCVI pg. 299. 496. 
oder geringere Zahl und Tiefe der Athembewegungen auf die Energie der thierischen Oxydationsprocesse keinen wesentlichen Einfluss ausüben werde. Für diesen Schluss war allerdings die von Dr. Wilhelm Dohmen ${ }^{1}$ ) in meinem Laboratorium (1863) festgestellte Thatsache von Belang, dass die Luftvolumina, welche ein in reinem Sauerstoff befindliches Thier einathmet, nur sehr wenig geringer sind, als diejenigen, die es in atmosphärischer Luft seinen Lungen zuführt. Obwohl also bei Athmung in reinem Sauerstoff den Lungen 4 bis 5 mal mehr von diesem Gase als gewöhnlich geboten wird, bleiben die Oxydationsprocesse doch unverändert. Zum tieferen Verständniss bleibt ferner zu beachten, dass nach meinen Blutgasanalysen $^{2}$ ) bei Athmung atmosphärischer Luft das arterielle Blut nahezu mit Sauerstoff gesättigt ist, also fast ebenso viel enthält, als ob das Thier reines Sauerstoffgas athme.

Eine Verstärkung der Athembewegungen konnte also schwerlich eine Steigerung des Stoffwechels deshalb bedingen, weil den Lungen mehr Sauerstoff zugeführt wurde. Die etwas grössere Arbeit der Athemmuskeln ist zu geringfügig, als dass sie wesentlich in Betracht kommen kann. Eine Möglichkeit, weshalb eine Verstärkung der Athembewegungen den Stoffwechsel steigern könnte, würde in der Verarmung des Blutes und der Gewebe an Kohlensäure gesucht werden dürfen. Denn die stärkere Lungenventilation bringt ja, wie ich gezeigt habe, wegen der stärkeren Ausscheidung der Kohlensäure, eine bedeutende Abnahme dieses Gases im Blute hervor. Es scheint aber nach einigen Thatsachen, als ob die Kohlensäure die Oxydationsprocesse in den Geweben mehr oder weniger behindere. Eine andere Möglichkeit könnte vielleicht darin gesucht werden, dass bei reichlicherer Zufuhr von Sauerstoff zu dem Blute die Reizbarkeit also auch die Reizungsgrösse des Nervensystemes - wie in der Apnoë-abnehme, was eine Verringerung des Sauerstoffverbrauches bedingen müsste. Die experimentelle Erledigung der Frage durch mich ${ }^{3}$ ), Dr. Dittmar Finkler und Dr. Ernst

1) Wilhelm Dohmen, Arbeiten des Bonner physiologischen Instituts 1865 .

2) E. Pflüger, Ueber die Ursache der Athembewegungen sowie der Dyspnoë und Apnoë. Diess Archiv. Bd. 1. 1868. pg. 69.

3) E. Pfl üger, Veber den Einfluss der Athemmechanik auf den Stoffwechsel. Diess Archiv. Bd. XIV. pg. 1. 
Prof. C. Voit u. d. Beziehung d. Athembewegungen zu dem Stoffwechsel. 633

Oertmann ${ }^{1}$ ) bestätigte meine Ansicht, dass die Athembewegungen keinen Einfluss auf den Stoffwechsel ausüben.

So wenig war man über diese Verhältnisse allgemein im Klaren, dass aus den Jahren 1866 und 1869 zwei umfángreiche Untersuchungen vorliegen, von denen die eine von Lossen, die andere von Eugen Berg ausgefiuhrt und in denen nirgends eine Ahnung von der wahren Beziehung zwischen Athembewegungen und Stoffwechsel zu finden ist, wie ich dies eingehend in dem ersten Aufsatze dieses Bandes des Archives bewiesen habe.

In einer 1870 erschienenen Polemik ${ }^{2}$ ) gegen Eugen Berg sucht L ossen seine 1866 veröffentlichten Resultate aufrecht zu erhalten und erklärt ausdrücklich, dass er alle Untersuchungen über den Einfluss der Zahl und T'iefe der Athembewegungen auf die Ausscheidung der Kohlensäure gemeinsam mit seinem Lehrer Prof. Carl Voit in dessen Laboratorium angestellt habe. In dieser also von V oit-Lossen gemachten Arbeit ist aber bekanntlich der Beweis zu liefern versucht worden, dass die Zahl und Tiefe der Athembewegungen die $\mathrm{Koh}$ lensäurebildung allerdings zu steigern oder zu verringern vermöge. Da nun jene Abhandlungen $H$. Lossen's in der von Voit herausgegebenen Zeitschrift für Biologie enthaiten sind, so kann man nicht zweifeln, dass Voit im Jahre 1870 noch leine richtige Vorstellung von der wahren Beziehung der Athembewegungen zu dem Stoffwechsel besass.

Ich hatte aber bereits im Jahre 1868 Beweise $^{3}$ ) für die Ursache der Athembewegungen, der Dyspnoë und Apnoë gegeben und gezeigt, dass die Verringerung des Sauerstoffs ohne Vermehrung der Kohlensäure und die Vermehrung der Kohlensäure ohne Verringerung des Sauerstoffs im Blute die Athembewegungen errege. Auf Grund Jahre lang fortgesetzter experimenteller Forschungen

1) Dr. Dittmar Finkler und Dr. Ernst Oertmann, Ueber den Einfluss der Athemmechanik auf den Stoffwechsel. Diess Archiv. Bd. XIV.pg. 38.

2) Dr. H. Lossen, Bemerkung zu der Abhandlung von Dr. Eugen Berg über den Einfluss der Zahl und Tiefe der Athembewegungen auf die Ausscheidung der Kohlensäure durch die Lunge. Zeitschrift für Biologie (1870) Bd. 6. pg. 298. - Berg's Dissertation findet sich auch im Deutschen Archiv f. klin. Medicin 1869. Bd. 6 pg. 291. -

3) Diess Archiv I. pg. 61 u. figde. 
hob ich hervor, dass die Verringerung des Sauerstoffs im Blute durch den Verbrauch der Gewebe bedingt sei, weshalb man die Sauerstoffaufnahme in den Lungen als das Secundäre, den Verbrauch in den Organen als das Ursächliche zu betrachten habe. Als Beleg citire ich wörtlich zwei Stellen aus meinen 1868 und 1869 erschienenen Aufsätzen :

„Das Blut findet nun, welches anch sein Gasgehalt sei und dies ist ein sehr wichtiger Punkt - die nöthige Zeit in den Lungen um sich mit Sauerstoff zu sättigen. So bedingt vermehrter Verbranch in den Capillaren vermehrte Aufnahme in den Congen.6 ${ }^{6}$ )

$E_{S}$ ist vielleicht nicht überflüssig, noch eine andere Stelle aus meiner 1869 publicirten Abhandlung über die Gase der Secrete hierher zu setzen. Der folgende Passus enthält natürlich noch die alte Auffassung über die Ursache der Zersetzung des Oxyhaemoglobins, was aber hier unwesentlich ist.

"Mir scheint es indessen unzweifelhaft, dass in dem lebenswarmen Blute immer freier Sauerstoff ist und nicht gelockert $\mathrm{zu}$ werden braucht. Denn das Oxyhaemoglobin zersetzt sich sofort und setzt Sauerstoff in Freiheit, sowie es in einem sauerstofffreien Raume sich befindet. Darauf beruht das Geheimniss des Abströmens des Sauerstoffes aus den Capillaren nach den Geweben, die continuirlich Sanerstoff in feste Verbindungen überfiuhren. Sobald die Tension des freien Sauerstoffs auf diese Weise gesunken ist unter eine gewisse Grenze, zerlegt sich neues Oxyhaemoglobin, so dass die Quantität von den Blutkörperchen gelieferten Sauerstoffs proportional dem Verbrauch der Gewebe eo ipso steigt. Umgekehrt versteht man so, warum in den Lungen das Blutkörperchen Sauerstoff continuirlich anzieht, weil nämlich bei der beträchtlichen Partiardichte des Sauerstoffs in der atmosphärischen Luft das Plama sanguinis so viel oder mehr Sauerstoff absorbirt, als nothwendig ist, um die Entstehung von Oxyhaemoglobin bei der Bluttemperatur zu ermöglichen. (2) $^{2}$

Bereits 1868 - 1869 wusste ich auf Grund meiner Analysen der Gase der Secrete, dass der Sauerstoffgehalt derselben fast von

1) Diess. Archiv. I. pg. 76 .

2) E. Pflüger, Die Gase der Secrete. Diesses Archiv. Bd. II. 1869. pg. 169 . 
Prof. C. Voit u. d. Beziehung d. Athembewegungen zu dem Stoffwechsel. 635

einerlei Ordnung mit den Beobachtungsfehlern oder ganz gleich Null sei. Daraus liess sich der Schluss ziehen, dass der Gehalt der lebendigen Zellen der Organe an freiem Sauerstoff sebr gering sein müsse. Vorher hatte schon L. Hermann ${ }^{1}$ ) behauptet, dass der lebendige Muskel keinen freien Sauerstoff enthalte. Der Muskel hat indessen bei Hermann's Methode vom Augenblick der Unterbrechung des Blutkreislaufs an hinreichend Zeit, den in ihm ursprünglich etwa enthaltenen freien Sauerstoff zu verbrauchen, ehe dieser durch Auspumpen gewonnen werden kann. Ich scbloss also, dass der Sauerstoff fortwährend aus dem Blute nach den Zellen der Organe wandern müsse, die so wenig enthalten, weil sie ihn fortwährend verbrauchen. Seitdem habe ich fortgefahren, immer nene Argumente für die Gewebeathmung beizubringen.

Der Satz, dass das Blut in den Lungen um so mehr Sauerstoff aufnimmt, je mehr im Organismus verbraucht wird, basirt auf meinen zahlreichen Blutgasanalysen, welche darthun, dass das Blut in den Lungen bei Athmung atmosphärischer Luft - vorausgesetzt nicht zu starke Reduction - sich immer nahezu sättigt.

Ich habe seit lange die respiratorischen Beziehungen der einzelnen Bestandtheile des Organismus zu einander durch Messung des Gasgehaltes und der Gasspannungen zu ermitteln gesucht oder ermitteln lassen. Voit hat stets nur die Beziehung des Gesanmtthieres zur atmosphärischen Luft erforscht und wie ich noch zeigen werde, nicht eine einzige Thatsache gefunden, aus der sich ein Schluss auf die Natur der Beziehung der oxydablen lebendigen Materie zu dem atmosphärischen Sauerstoff machen liesse.

Seitdem ich in diese Verhältnisse Klarheit brachte, finde ich öfter bei Anderen mit den meinigen identische Erörterungen über die Natur der respiratorischen Beziehungen der einzelnen Organe zu einander, ohne dass die betreffenden Autoren mit einer Andeutung meiner erwähnten.

$\mathrm{Zu}$ diesen Herren gehört Carl Voit. Ganz urplötzlich, ohne mit einer Silbe der in Lossen's Arbeit niedergelegten Resultate mehr zu gedenken, die er doch gemeinsam mit Lossen als dessen Lehrer zu Tage gefördert hat, spricht sich Voit - erst 1870 -

1) Ludimar Hermann, Untersuchungen über den Stoffwechsel der Muskeln. Berlin 1867. pg. 27. 
in seiner Zeitschrift für Biologie genau so aus, wie ich zwei Jahre vor ihm, im Gegensatz zu den bei Lossen's und seinen Experimenten gemachten Erörterungen und mit vollkommenem Stillschweigen über die Quelle seiner besseren Erkenntniss, mag diese nun in meinen Abhandlungen oder sonst wo anders liegen.

Diese richtige Auffassung Voit's findet sich zuerst in der gegen Liebig gerichteten, 1870 erschienenen, 98 Seiten langen $\mathrm{Ab}$ handlung1): "Ueber die Entwicklung der Lehre von der Quelle der Muskelkraft und einiger Theile der Ernährung « auf Seite $389 \mathrm{u}$. flg.

Die hier in Betracht kommende Stelle lantet: "Das dem Sauerstoffvorrath der Organe Entzogene wird aus dem an das Haemoglobin des Blutes gebundenen Sauerstoff wieder ergänzt und dieser wird endlich in der Lunge durch neuen aus der eingeathmeten Luft ersetzt. Der letztere Ersatz richtet sich also nach dem Verbranch in den Organen; die Zahl der Athemzüge ist das Bedingte und nicht das Bedingende. Ist in den Organen mehr zersetzt und mehr Sauerstoff verbraucht worden, so hat in Folge davon der Sauerstoffgehalt des Blutes abgenommen und sein Kohlensäuregehalt zugenommen und nun werden dadurch die Regulatoren der Athmung in dem verlängerten Mark zu häufigeren und tieferen Athemzügen gezwungen, um neuen Sauerstoff zuzuführen und die überschüssige Kohlensäure wegzubringen."

Damit über Voit's Auffassung kein Zweifel bleibe, will ich noch eine andere Stelle ${ }^{2}$ ) seiner 1872 erschienenen Abhandlung "Ueber die Bedeutung des Leimes bei der Ernährung " hierher setzen.

"Die Fette und Kohlehydrate nannte Liebig die respiratorischen Nahrungsmittel. Er sagte, ihre Zersetzung habe einen andern Grund als die des Eiweises, welches als organisirtes durch die Arbeit zerfällt, sie verbrennen einfach durch den in Folge der Respirationsbewegungen in das Blut aufgenommenen Sauerstoff. Man stellte sich vor, der in dem Körper aufgenommene Sauerstoff sei der Zerstörer und er nage so lange an den Fetten und Kohlehydraten, bis er ganz in Beschlag genommen sei. Die Grösse der

1) Carl Voit, Ueber die Entwicklung der Lehre von der Quelle der Muskelkraft etc. Zeitschr. f. Biologie, pg. 388 u. ff.

2) Carl Voit, Ueber die Bedeutung des Leimes bei der Ernāhrung, Zeitschr. für Biologie. Bd. VIII. (1872). pg. 382 etc. 
Prof. C. Voit u. d. Beziehung d. Athembewegungen zu dem Stoffwechsel. 637

Aufnahme des Sauerstoffs Jiess man abhängen von der Zahl und Tiefe der Athemzüge; wenn es aussen kalt ist, so brauchen wir darnach nur durch häufigere und tiefere Athembewegungen mehr Sauerstoff einzupumpen um mehr von den Respirationsmitteln zu verbrennen und so dem Körper die genügende Wärme zu liefern.

"Der Sauerstoff ist aber nicht die nächste Wärme der Zersetzung von Substanzen im Körper, sondern sie zerfallen darin nach und nach in immer einfachere Producte, die dann allmählig Sauerstoff aufnehmen. Ich habe dies für die Eiweisszersetzung schon lange ausgesprochen, da dieselbe in keinem Verhältnisse steht zur Sauerstoffaufnahme; das Eiweiss kann ja schon in dem für Oxydationen gewiss ungünstigen Darm den Zerfall in Leucin etc. beginnen, wie er auch im übrigen Körper stattfindet. Ebenso ist es für die Fette und Kohlehydrate; die Ursachen ihres Zerfalls sind ganz andere. Ich habe schon bei einer anderen Gelegenheit (Hier citirt er Biologie von 1871. Ref.) "hervorgehoben, dass bei den gewöhnlichen Verbrenuungsprocessen z. B. des Holzes der Sauerstoff auch nicht die Ursache des Zerfalles ist, sondern die Anzündungstemperatur, welche die Zerlegung in niedere Produkte bedingt, die bei ihrem allmähligen weiteren Zerfall Sauerstoff binden; letztere ist nur von Einfluss auf die beim Zerfall entstehenden Produkte.

"Wenn der Sauerstoff nicht das einleitende Moment für die Stoffzerlegungen im Thierkörper ist, so können letztere auch nicht von der Zahl und Tiefe der Athemzüge abhängen. Ich habe schon mehrmals" (hier citirt er wieder die Biologie von 1870 und 1871), "darauf aufmerksam gemacht, dass die Athemzüge nicht direkt die Zersetzung im Körper reguliren, sondern dass primär in den Geweben dieStoffe nach bestimmten Regeln, unabhängig vom Sauerstoff, zerfallen und dann, indem die weitern Produkte sauerstoffreicher werden, Sauerstoff aus dem Blute weggenommen und Kohlensäure dahin abgegeben wird, was secundär durch Erregung der Regulatoren im verlängerten Marke Athembewegungen nach sich zieht, durch welche der aus dem Blute genommene Sauerstoff wieder ergänzt und die Kohlensäure entfernt wird.

"Der letztere Ersatz richtet sich also nach dem Verbrauch in den Organen; würde in den Geweben durch die innere Athmung kein Sauerstoff verbraucht, so würden die heftigsten Athembewegungen keinen weiteren Sauerstoff ins Blut bringen." 
Ich bemerke, dass die Sperrung einiger Worte in dem Citate nicht im Originaltext vorhanden ist, sondern von mir herrührt.

Es ist also nicht zu bestreiten, dass ich in den oben citirten Abhandlungen zwei Jahre vor Voit, d. h. 1868, mit der grössten Bestimmtheit die Athembewegungen durch die Verringerung des Blutsauerstoffs und die Vermehrung der Blutkohlensäure, und ferner die Verringerung des Blutsauerstoffs und die Vermehrung der Blutkohlensäure durch die lebendigen Zellen der Organe bedingt sein liess, sodass jch den Ausdruck branchen durfte: die Organe sind die Ursache des Sauerstoffverbrauchs des Blutes und dieser Verbrauch ist die Ursache ${ }^{1}$ ) der Sauerstoffa ufnahme in den Lungen, wo das Blut um so mehr aufnimmt, je mehr es verloren hat, weil es sich sättigt.

Wenn man die aus dem Voit'schen Laboratorium hervorgegangenen Publicationen zu Grunde legt, muss man zu der Ansicht gelangen, dass sich der Umschwung in Voit's Anschauungen im Laufe des Jahres 1870 vollzogen hat. Denn im Heft II des Bandes VI der Biologie von 1870 vertheidigt Voit's Schüler H. Lossen seine Untersuchungen gegen Eugen Berg und hält sie aufrecht, wiewohl der Einfluss der Athemmechanik auf die Kohlensäurebildung nicht mehr betont, aber auch nicht widerrufen wird. In dem unmittelbar darauf folgenden Hefte III desselben Bandes VI der Biologie von 1870 entwickelt Voit nun plötzlich die richtigen, durch mich zwei Jahre früher festgestellten Ansichten zum ersten Male, wie ich es oben dargelegt habe. Sein Aufsatz folgt in dem Bande dem von Lossen unmittelbar nach; und es ist nicht uninteressant zu sehen, wie er sich in die neue Vorstellung noch nicht vollständig genug eingearbeitet hat, sodass im Anfange der langen gegen Liebig gerichteten Abhandlung ein Passus vorkommt, wo er in seine alte, irrige Auffassung zurückfällt. Dieser Passus ${ }^{2}$ ) heisst: "Ich selbst habe am Hunde in meinen ersten 24 stündigen Versuchen bei Hunger und namentlich bei reichlicher Fleischnahrung ein kleines Plus von Harnstoff bei der Arbeit gefunden; den Grund

1) Ich bin mir wohl bewusst, dass hier und an anderen Stellen das Wort "Ursachec in einer abgeleiteten Bedeutung gebraucht ist. Denn Ursache ist die Potenz, welche die Wirkung ganz allein ohne Beihülfe anderer Potenzen hervorruft.

2) Carl Voit, Ueber die Entwicklung der Lehre von der Quelle der Muskelkraft in Zeitschr. f. Biologie. Bd. 6. - 1870. - pg. 336. 
der geringen Vermehrung des Eiweissumsatzes suchte ich zum Theil in der grösseren Wasseraufnahme und Harnmenge, vorzüglich aber in dem durch die verstärkten Herz-; Athem- und Körperbewegungen mehr aufgenommenen Sauerstoff (!), der dann bei Mangel an Fett oder Kohlehydraten mehr Eiweiss des intermediären Säftestroms angreift."

Heisst diess nun nicht unzweifelhaft, dass mehr oxydirt wurde, weil die verstärkte Athmung dem Blute und den Organen mehr Sauerstoff zuführe? Hier ist es aber keineswegs seine Meinung, dass er eine alte überlebte Ansicht von sich mittheile. Denn er fährt fort: wich habe daher damals schon gesagt: würde man den überschüssigen (!!) Sauerstoff durch einen anderen Stoff, der sich damit verbindet, z. B. durch Fettzusatz in Beschlag nehmen, so könnte man die grössere Eiweisszersetzung völlig aufheben. In der That hat sich dies auch beim Menschen, wenn er nicht mager war oder stickstoffreie Kost in der Nahrung in genügender Menge erhielt, so herausgestellt. Es kann aber auch beim Menschen, wie beim Hunde, unter gewissen Umständen eine kleine Steigerung der Eiweisszersetzung eintreten, namentlich bei fettarmer und für die Arbeit ungenügender Zufuhr stickstofffreier Substanzen oder bei sehr reichlicher (!) Sauerstoffaufnahme bei angestrengter Thätigkeit.“

Dies steht in demselben Aufsatz, in dem später behauptet wird, dass der Sauerstoffreichthum gar keinen Einfluss auf die Grösse der Oxydation hat, wie dies der Leser aus den oben ausgezogenen Stellen desselben Voit'schen Aufsatzes sich erinnern wird. Dieses Zurückfallen Voit's in die alte unrichtige Ansicht am Anfange des Aufsatzes ist ausserordentlich merkwürdig, wenn man - da Voit sich als Original anführt - die Annahme machen muss, dass er durch eigene Gedankenarbeit und eigene Erfahrungen sich zu der richtigen Ansicht hindurchgearbeitet, die er der Welt in demselben Aufsatze mitzutheilen sich entschlossen hat.

Im nächsten Jahre 1871 sucht Voit ${ }^{1}$ ) in einer mit Pettenk ofe $r$ an dem grossen Respirationsapparat gemeinsam unternommenen Arbeit, die aber in Wirklichkeit schon einmal im Anfange der sech-

1) Max von Pettenkofer und C. Voit, Ueber die Zersetzungsvorgänge im Thierkörper bei Fütterung mit Fleisch. Zeitschrift für Biologie 1871. Bd. 7. pg. 455 . 
ziger Jahre in abgekürzter Form publicirt worden war, Beweise für die Ansicht beizubringen, dass die Sauerstoffaufnahme in den Lungen eine secundäre, durch die oxydirbare Substanz in den Organen bedingte Erscheinung sei. Es könnte mir nur angenehm sein, wenn $V_{0}$ it neue Beweise für meine Ansicht beigebracht hätte.

Ich will hier davon absehen, dass die mit dem Respirationsapparat Pettenkofer's erhaltenen Zahlenwerthe für den Sauerstoff, das Transpirationswasser, das Sumpfgas und den Wasserstoff mit wahrhaft riesigen Fehlern behaftet sind, wofür ich die Beweise in Händen habe; ich will absehen von den meist unzulässigen Voraussetzungen, auf Grund deren ihre Stoffwechselrechnungen ausgeführt sind; ich will absehen von ihren Controlrechnungen, in denen sie die Rechnung, welche controlirt werden soll, einfach in anderer Form wiederholen und darin eine Bestätigung ihrer Voraussetzungen zu erkennen glauben.

Sehen wir also für jetzt von Alledem hier $a b$, und gehen wir zu dem von M. v. Pettenkofer und Carl Voit vorgebrachten Beweise über.

In einer vom 16. Februar bis 9. März 1863 an einem Hunde von 33 Kilo durchgeführten Versuchsserie, in welcher das Thier täglich 1500 Gr. mageres Fleisch erhielt und 5 mal (16. Februar, 20. Febr., 23. Febr., 27. Febr., 4. März) im Respirationsapparat auf die Gesammtmenge seiner Einnalimen und Ausgaben untersucht wurde, glauben Voit und Pettenkofer auf Grund ihrer Analysen und Rechnungen gefunden zu haben, dass der Hund im Anfange der Serie mehr Fett und weniger Fleisch, später kein Fett, aber grössere Fleischmengen in seinem Körper oxydirte. Daraus würde also folgen, dass wegen des eiweissreichen und fettarmen Futters eine Aenderung in dem Zustande des Thieres eingetreten ist. Dieser Aenderung entsprechend ergab sich auch, dass der Hund im Anfange der Serie etwas mehr Sauerstoff in 24 Stunden absorbirte als später.

Daraus schliessen sie dann, dass die Grösse, Art und Natur der Zersetzung im Körper die Sauerstoffaufnahme in den Lungen regulire.

Ihre Argumentation ${ }^{1}$ ) ist genauer folgende:

1) Max von Pettenkofer und Carl Voit, Ueber die Zersetzungsvorgänge im Thierkörper bei Fütterung mit Fleisch in Zeitschr. für Biologie 1871. Bd. 7. pg. 454. 
Prof. C. Voit u. d. Beziehung d. Athembewegungen zu dem Stoffwechsel. 641

"Aus der ( (am Anfang und Ende der Versuchsserie verschiedenen, Ref.) "Fleisch- und Fettzersetzung werden nun die sonst unbegreiflich scheinenden Verhältnisse der Sauerstoffaufnahme und der Kohlensäureausscheidung leicht erklärlich; die beiden nehmen nämlich - - stetig ab. « - Folgen unwesentliche weitere Erörterungen und dann geht es weiter:

"Die hier beobachtete Abuahme in der Sauerstoffaufnahme zeigt aufs Deutlichste an, dass die letztere nicht das primäre ist, $d . h$. dass der Sauerstoff nicht je nach dem Athemmechanismus ins Blut eintritt and dann seine verheerenden Wirkungen beginnt und so lange zerstört, bis er verbraucht ist, sondern dass vielmehr im Thierkörper die Stoffe, z. B. Eiweiss oder Fett, je nach den Bedingungen in den Organen zerfallen und erst bei dem fortschreitenden Zerfall so viel Sauerstoff aus dem Blute aufnehmen als sie nöthig haben, um sich in Kohlensäure und Wasser etc. zu verwandeln; in Folge dieser Wegnahme von Sauerstoff aus dem Blute kann dann neuer von Aussen ins Blut wieder eintreten. (1)

Es ist nicht schwer, den Fehler in diesen Schlussfolgerungen zu finden.

Der Hund hatte in Folge einer veränderten länger fortgesetzten Diät, die reich an Albuminaten und sehr arm an Fetten und Kohlehydraten war, allmählig eine Veränderung in der Zusammensetzung und in Folge dessen auch der Processe in seinem Körper erfahren. Diese Veränderung der Zusammensetzung des Thierkörpers bedingte auch eine Veränderung des Sauerstoffverbrauches und es ist allerdings unzweifelhaft, dass der Sauerstoffverbrauch hier als das Bedingte, die veränderte Zusammensetzung des Thierkörpers als das ursächliche Moment erscheint. - Dasselbe lehrt aber auch die Vergleichung des relativ kleinen Sauerstoffverbrauchs eines Elephanten mit dem relativ sehr grossen einer Maus, ohne dass diess uns berechtigte, die Sauerstoffaufnahme in den Lungen, sowie die Athembewegungen als das Bedingte, die zersetzbare Substanz des Körpers als das Bedingende und Ursächliche $\mathrm{zu}$ betrachten, was ja auch ausser Voit noch Niemand versucht hat.

Wenn also Max von Pettenkofer und Carl Voit aus der am Anfang und Ende der Versuchsserie verschiedenen Fleisch- und

1) Max v. Pettenkofer und Carl Voit, a. a. O. pg. 455.

E. Püüger, Archiv f. Physiologie. Bd. XIV. 
Fettzersetzung schliessen, dass die oxydirbare Substanz und nicht der Sauerstoff die primäre und letzte Ursache des Sauerstoffverbrauches und in Folge dessen der veränderten Athembewegungen sei, so machen sie bierbei die stillschweigende und geradezu unzulässige Voraussetzung, dass der Sauerstoff im veränderten Thierkörper quantitativ und qualitativ (siche Anmerkung) unverändert geblieben sei. Da aber bei Veränderung der Zusammensetzung des Thierkörpers das Blut nicht als unverändert angenommen werden darf, also auch nicht der Sauerstoffgehalt desselben, da ferner bei veränderter Erregbarkeit des A themcentrums im verlängerten Marke auch die Athembewegungen nicht als constante Grösse betrachtet werden dürfen, so ist es gewiss, dass alle bei der Grösse der Oxydation im Organismus betheiligten Faktoren geändert sind: Athembewegungen, Sauerstoffgehalt des Körpers und oxydirbare Substanz. $\mathrm{Ob}$ und wie viel die Aenderung des einzelnen Faktors die Grösse der Verbrennung beeinflusst, lässt sich also nicht entscheiden.

Nachdem bis dahin Voit an keiner Stelle auch nur angedeutet hat, dass ich längst vor ihm die Natur der Beziehungen der Sauerstoffabsorption in den Lungen zu den Oxydationsprocessen in den Capillaren dargelegt habe, erklärt er endlich 1872 im Bande 8 der Biologie, dass er erfreut sei, "in dieser "seiner" Anschaung in Pflüger einen Bundesgenossen erhalten zu haben, der auf ganz anderem Wege zu der gleichen Vorstellung gelangt " sei.") Voit citirt hier meine Abhandlung wüber die Diffusion des Sauerstoffs etc. von 1872 , nicht aber die von 1868 und 1869. Er hebt besonders hervor, wie er nschon mehrmals darauf aufmerksam gemacht, dass die Athemzüge nicht direct die Zersetzung im Körper reguliren und giebt zugleich alle Stellen seiner Schriften an, wo diese neue Lehre von ihm zu finden sei: d. i.

Biologie 1870 pg. 388 u. 1871 pg. 197 u. 494.

Ich habe aus der Zeitschrift für Biologie alle Stellen ausgezogen, wo derselbe Gedanke von Voit ausgesprochen worden ist: 1870. Band 6. pg. 388 .

Anmerkung. Ich denke hier an die noch nicht sicher widerlegte, in den Geweben erzeugte Verwandlung von neutralem Sauerstoff in Ozon.

1) Carl Voit, Ueber die Bedeutung des Leimes bei der Ernährung. Zeitschr. für Biologie 1872. Bd. 8. pg. 384 . 
Prof. C. Voit u. d. Beziehung d. Athembewegungen zu dem Stoffwechsel. 643

1871. Band 7. pg. 197. - Band 7. pg. 454. - Band 7. pg. 465. Band 7. pg. 495.

1872. Band 8. pg. 383 und 384. -

1873. Band 9. pg. 32. - Band 9. pg. 509. - Band 9. pg. 528.

1874. Band 10. pg. 230. -

An allen Stellen steht fast genau Dasselbe, oft mit fast denselben Worten und grosser Ausführlichkeit.

Zur allgemeinen Orientirung nur die Bemerkung, dass die Erkenntniss der Abhängigkeit und Bedingtheit der Athembewegungen durch den allgemeinen Stoffwechsel, welche Voit sich zuschreibt, in der That doch nur der veränderte Ausdruck der alten längst bekannten Wahrheit ist, dass die Venosität des Blutes die Athembewegungen errege, was weder Voit noch ich entdeckt haben. Es kann sich also nur noch um die Ursache des Venöswerdens des Blutes handeln, d. h., um die Natur der Beziehung des Sanerstoffs zu der oxydirbaren Substanz. Obwohl fast alle Physiologen heute mit mir die Ansicht theilen, dass der Sauerstoff nicht die erste Ursache der thierischen Verbrennung sei, will ich doch nicht unterlassen, hier auszusprechen, dass die von der Gegenwart des Sauerstoffs unabhängige Bildung der Kohlensäure mit absoluter Strenge für die thierischen Körper bis heute von Niemand, auch von mir nicht bewiesen ist. An dieser fundamentalen, aber sehr schwierigen . Frage arbeite ich seit langer Zeit bis auf den heutigen Tag, und so viel ist gewiss, dass ich und meine Schüler eine Reihe wichtiger Argumente für die indirecte Oxydation der thierischen Gewebe beigebracht haben - Argumente, welche auch bei den Fachgenossen eine wohlwollende Anerkennung fanden. Der naufgespeicherte sauerstoff von C. Voit und Max von Pettenkofer aber hat, wie ich beweisen kann und werde, mit der Physiologie Nichts, mit der Methode Pettenkofer's Alles zu thun.

Gerade 'weil ich der Ansicht war, dass die Unabhängigkeit der Oxydationsprocesse resp. Kohlensäurebildung von dem Partiardrucke des freien Sauerstoffs noch nicht über allen Widerspruch erhaben ist, habe ich mit den Herren Dr. Dittmar Finkler und Dr. Ernst Oertmann die Athembewegungen resp. Lungenventilation variirt und gleichzeitig die Grösse der Oxydationsprocesse gemessen. 
Ich hielt anfänglich eine Vertheidigung gegen die Reclamationen Voit's und des ihm secundirenden Dr. Siegfried Wolffberg nicht für nothwendig, da die Jedem zugängliche neuere Literatur ja das Urtheil der Sachverständigen ohne mein Zuthun bestimmen muss. Nachdem aber V o i t's Reclamationen wie etwas Berechtigtes auch in den Jahresbericht übergegangen sind, glaubte ich nicht länger schweigen zu dürfen.

Meine Vertheidigung soll auch hiermit nicht abgeschlossen sein; ich werde vielmehr eingehender zu den noch nicht erledigten Reclamationen Voit's an einer anderen Stelle zurückkehren.

Um die Beschwerde V o it's wegen mangelnder Berücksichtigung seiner Arbeiten durch mich richtig würdigen zu können, muss ich einige Thatsachen anführen, über welche ich sonst nie gesprochen hätte und seit Jahren geschwiegen habe.

Vo it hat in seinen zahlreichen Abhandlungen bei wiederholter directer Besprechung verschiedener unter meiner Leitung and Mitwirkung ausgeführten Arbeiten mich immer von jeher absolut ignorirt.

$V_{0}$ i t hat von meinen Untersuchungen über Respiration absolut niemals Notiz genommen, so zwar dass in seinen gesammten Schriften mit Einschluss derjenigen über Respiration mein Name auch nicht ein Einziges Mal erwähnt wird, auch dann nicht, wenn er Thatsachen und Gedanken benutzt, welche mein Eigenthum sind. Dies fällt um so schwerer in's Gewicht, als Voit die Literatur des Gegenstandes, den er behandelt, sonst recht genau zu berücksichtigen pflegt.

Voit hat erst vom Jahre 1872 ab mehrmals sich auf mich bezogen, aber ausschliesslich und nur dann, wenn er Entdeckungen und Gedanken von mir sich aneignen will, auf welche er nicht das geringste Anrecht hat. Es ändert an der Sache nichts, wenn er dazu die höfliche Form wählt, mich als Bundesgenossen "seiner" Ansichten zu begrüssen.

Ich werde also von ihm (resp. Voit - W o lffberg) absolut nur dann erwähnt, wenn ich geschädigt werden soll.

Erstaunt war ich, dass derselbe Gelehrte über mangelnde Berücksichtigung seiner Arbeiten durch mich sich zu beschweren keinen Anstand nimmt, obwohl ich ihn trotz alledem doch öfter, als ich vielleicht verantworten kann, als Gewährsmann citirt und niemals durch irgend eine Bemerkung verletzt habe. 